Proc. of the 11 Int. School on Theoretical Physics Symmetry and Structural Properties of Condensed Matter, Rzeszów 2014

\title{
Combinatorics of Lax Objects in Bethe Ansatz
}

\author{
R. STAGRACZYŃSKI* \\ Rzeszów University of Technology, The Faculty of Mathematics and Applied Physics, \\ Al. Powstańców Warszawy 6, 35-959 Rzeszów, Poland
}

\begin{abstract}
Algebraic Bethe Ansatz, also known as quantum inverse scattering method, is a consistent tool based on the Yang-Baxter equation which allows to construct Bethe Ansatz exact solutions. One of the most important objects in algebraic Bethe Ansatz is a monodromy matrix $\widehat{M}$, which is defined as an appropriate product of so-called Lax operators $\widehat{L}$ (local transition operators). Monodromy matrix as well as each of Lax operators acts in the tensor product of the quantum space $\mathcal{H}$ with an auxiliary space $\mathbb{C}^{2}$. Thus $\widehat{M}$, when written in the standard basis of auxiliary space, consists of four elements $\widehat{A}, \widehat{B}, \widehat{C}, \widehat{D}$, which are the operators acting in quantum space $\mathcal{H}$, where $\widehat{B}$ and $\widehat{C}$ are step operators and the remaining generate all constants of motion. In this work a consistent method of construction of the Bethe Ansatz eigenstates in terms of objects $\widehat{a}, \widehat{b}, \widehat{c}, \widehat{d}$ i.e. matrix elements of the Lax operators in the auxiliary space is proposed.
\end{abstract}

DOI: 10.12693/APhysPolA.128.216

PACS: 03.65.Aa, 75.10.Jm

\section{Introduction}

Let us consider the one dimensional Heisenberg XXX model. The set of nodes $\tilde{N}=\{j=1,2, \ldots, N\}$ is equally spread on a circle and each node is occupied by the spin $s=1 / 2$. Therefore, each magnetic configuration is described by the mapping $f: \tilde{N} \rightarrow \tilde{2}$, presented by

$$
\left|i_{1}, i_{2}, \ldots, i_{N}\right\rangle, i_{j} \in \widetilde{2} \text { for } j \in \widetilde{N} \text { where } \widetilde{2}:=\{-,+\} .(1)
$$

The set of all magnetic configurations of the form (1) constitutes the configuration space $Q$ of the system. The Hilbert space $\mathcal{H}$ of the model is spanned over the basis $Q$. Dynamics of the system is governed by the Hamilton operator

$$
\widehat{H}_{N}=J \sum_{j=1}^{N}\left(\widehat{\sigma}_{j}^{x} \widehat{\sigma}_{j+1}^{x}+\widehat{\sigma}_{j}^{y} \widehat{\sigma}_{j+1}^{y}+\widehat{\sigma}_{j}^{z} \widehat{\sigma}_{j+1}^{z}-I_{N}\right),
$$

where $J$ is the coupling constant, $\widehat{\sigma}^{i}$ are standard Pauli matrices, and

$$
\widehat{\sigma}_{j}^{i}=\widehat{I} \otimes \ldots \widehat{I} \otimes \underbrace{\widehat{\sigma}^{i}}_{j} \otimes \widehat{I} \ldots \otimes \widehat{I} .
$$

Bethe eigenstates of this Hamiltonian are parametrized by sets of the so-called spectral parameters $\left\{\lambda_{1}, \lambda_{2}, \ldots, \lambda_{r^{\prime}}\right\}$, and the latter are solutions of the Bethe Ansatz equations [1]

$$
\left(\frac{\lambda_{j}+i / 2}{\lambda_{j}-i / 2}\right)^{N}=\prod_{k \neq j}^{r^{\prime}} \frac{\lambda_{j}-\lambda_{k}+i}{\lambda_{j}-\lambda_{k}-i},
$$

where $\tilde{r}^{\prime}$ is the set of the Bethe pseudoparticles.

\section{Algebraic Bethe Ansatz for a magnetic ring}

One of the main ingredients of algebraic formulation of Bethe Ansatz is the Lax operator $\widehat{L}_{j, a}(\lambda)$ defined as:

\footnotetext{
*e-mail: rstag@prz.edu.pl
}

$$
\widehat{L}_{j, a}(\lambda)=\lambda \widehat{I}_{\mathcal{H}} \otimes \widehat{I}_{V}+i \sum_{\alpha} \widehat{s}_{j}^{\alpha} \otimes \widehat{\sigma}^{\alpha},
$$

where $\widehat{I}_{\mathcal{H}}$ and $\widehat{I}_{V}$ are unit operators in the physical and auxiliary space $[2,3]$. It can be written in a form

$$
\begin{gathered}
\widehat{L}_{j, a}(\lambda)=\left(\begin{array}{cc}
\lambda \widehat{I}+i \widehat{s}_{j}^{z} & \widehat{s}_{j} \\
\widehat{s}_{j}^{+} & \lambda \widehat{I}-i \widehat{s}_{j}^{z}
\end{array}\right)= \\
\left(\begin{array}{cc}
\widehat{a}_{j}(\lambda) & \widehat{b}_{j} \\
\widehat{c}_{j} & \widehat{d}_{j}(\lambda)
\end{array}\right),
\end{gathered}
$$

and the set of matrix elements of $\widehat{L}_{j, a}(\lambda)$ i.e. $\{\widehat{a}(\lambda), \widehat{b}, \widehat{c}, \widehat{d}(\lambda)\}$ will be referred to as that of the Lax objects. Construction of the Bethe vectors in algebraic Bethe Ansatz (ABA) requires monodromy matrix

$$
\widehat{M}(\lambda):=\widehat{L}_{N}(\lambda) \ldots \widehat{L}_{1}(\lambda)=\left(\begin{array}{cc}
\widehat{A}(\lambda) & \widehat{B}(\lambda) \\
\widehat{C}(\lambda) & \widehat{D}(\lambda)
\end{array}\right),
$$

where $\widehat{B}(\lambda)$ and $\widehat{C}(\lambda)$ are, respectively, the creation and annihilation operators for a magnon with the rapidity lambda when created from the ferromagnetic saturation state, whereas $\widehat{A}(\lambda)+\widehat{D}(\lambda)$ form the transfer matrix which yields, e.g., the Hamiltonian

$$
\widehat{H}=\left.\frac{\mathrm{i}}{2} \frac{\mathrm{d}}{\mathrm{d} \lambda} \ln (\widehat{A}(\lambda)+\widehat{D}(\lambda))\right|_{\lambda=i / 2}-\frac{N}{2} \widehat{I} .
$$

Any eigenvector can be written in terms of operator $\widehat{B}(\lambda)$ :

$$
\left|\lambda_{1} \lambda_{2} \ldots \lambda_{r^{\prime}}\right\rangle \cong \prod_{\alpha=1}^{r^{\prime}} \widehat{B}\left(\lambda_{\alpha}\right)|0\rangle,
$$

where spectral parameters $\tilde{\lambda}$ are solutions of $(3)$, and $|0\rangle$ is the vacuum state. Examples of such collections can be found in $[4-8]$.

\section{Combinatorics of Lax objects}

The dimension of the physical space $\mathcal{H}$ is $2^{N}$ and the dimension of $\mathcal{H} \otimes V$ is $2^{N+1}$ and both exponentially 
increases with the length of the spin chain. A way to overcome this problem comes from the fact that matrix of $\widehat{M}(\lambda)$ by its definition is sparse therefore it is less computational consuming to define it by its matrix elements from the set $\tilde{M}_{M}$

$$
\widehat{M}(\lambda)=\sum_{\widehat{m} \in M_{M}} \widehat{m}(\lambda),
$$

where

$$
\widehat{m}(\lambda)=\prod_{j \in \tilde{N}} \widehat{x}_{j}(\lambda)
$$

is a monomial of degree $N$, with factors

$$
\widehat{x}(\lambda) \in\{\widehat{a}(\lambda), \widehat{b}, \widehat{c}, \widehat{d}(\lambda)\} .
$$

This statement is supported by the fact that Lax objects act effectively only on the space of a single node

$$
\begin{aligned}
& \widehat{x}_{j}(\lambda)=\widehat{I}_{2} \otimes \ldots \otimes \widehat{x}(\lambda) \otimes \ldots \otimes \widehat{I}_{2}, \\
& \widehat{a}(\lambda)=\begin{array}{r|cc}
+ & p & 0 \\
- & 0 & q
\end{array} \quad \widehat{b}=\begin{array}{r|cc}
+ & 0 & 0 \\
- & \mathrm{i} & 0
\end{array} \\
& \widehat{c}=\begin{array}{c|cc} 
& + & - \\
\hline+ & 0 & \text { i } \\
- & 0 & 0
\end{array} \quad \widehat{d}(\lambda)=\begin{array}{r|cc} 
\\
\hline+ & q & 0 \\
- & 0 & p
\end{array},
\end{aligned}
$$

where $p=\lambda+\mathrm{i} / 2, q=\lambda-\mathrm{i} / 2$. The set of all monomials $\tilde{M}_{M}$ decomposes into subsets

$$
\tilde{M}_{M}=\tilde{M}_{A} \cup \tilde{M}_{B} \cup \tilde{M}_{C} \cup \tilde{M}_{D},
$$

thus each of operators $\widehat{A}(\lambda), \widehat{B}(\lambda), \widehat{C}(\lambda), \widehat{D}(\lambda)$ may be written as

$$
\widehat{X}(\lambda)=\sum_{\widehat{m} \in \tilde{M}_{X}} \widehat{m}(\lambda), \quad X \in\{A, B, C, D\} .
$$

A useful classification of monomials $m$ is provided by the weight $w(m)=\left(w_{a}, w_{b}, w_{c}, w_{d}\right)$ where $w_{x}$ is simply the number of the Lax object of type $x$ in $m$. Obviously

$$
w_{a}+w_{b}+w_{c}+w_{d}=N .
$$

In terms of Eq. (10), each monomial is a word of length $N$ composed from Lax objects, and formally number of all combinations is $4^{N}$. Important question is which of them are needed to construct ABA operators $\widehat{A}(\lambda), \widehat{B}(\lambda)$, $\widehat{C}(\lambda), \widehat{D}(\lambda)$. Elementary properties of those operators imply the following relations:

$$
\begin{aligned}
& w_{b}=w_{c}, \quad m \in \tilde{M}_{A} \text { or } m \in \tilde{M}_{D}, \\
& w_{b}=w_{c}+1, \quad m \in \tilde{M}_{B}, \\
& w_{c}=w_{b}+1, \quad m \in \tilde{M}_{C} .
\end{aligned}
$$

Final construction of monomials comes along with properties of Eq. (10) and matrices of the Lax objects. This procedure can be simply visualized by introducing a frame consisting of $2(N+1)$ points spread equally between two levels of Fig. 1, where levels 1 and 2 can be chosen from the set $\{p, q\}$. With respect to the scheme proposed in Fig. 1 each of the Lax objects is represented by the edge connecting points for adjacent $j$ 's, and each

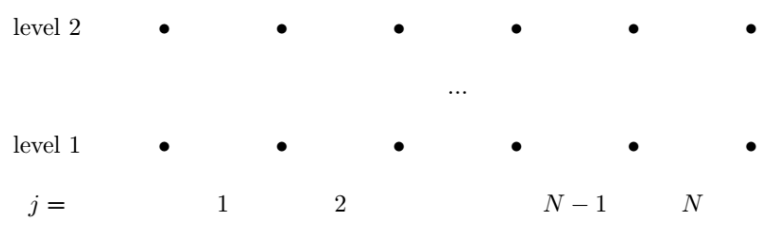

Fig. 1. The frame for graphic construction of monomials $\widehat{m}$.

monomial can be viewed as a quasi-continuous path without branches. Figure 2 presents one of possible conventions of choosing levels 1 and 2 .

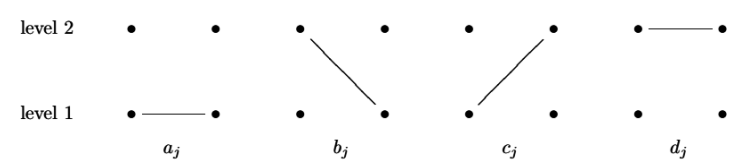

Fig. 2. Convention of graphic representation of the Lax objects $\widehat{a}, \widehat{b}, \widehat{c}, \widehat{d}$. Within this convention, if a path terminates after the first $j$ steps at the level 1(2), then the next step can be either $a$ or $c(b$ or $d)$, in accordance with the multiplication rule of consecutive Lax operators.

According to this choice, each of monomials belonging to operators $\widehat{A}(\lambda), \widehat{B}(\lambda), \widehat{C}(\lambda), \widehat{D}(\lambda)$ is represented by a unique path of the shape from Fig. 3 .

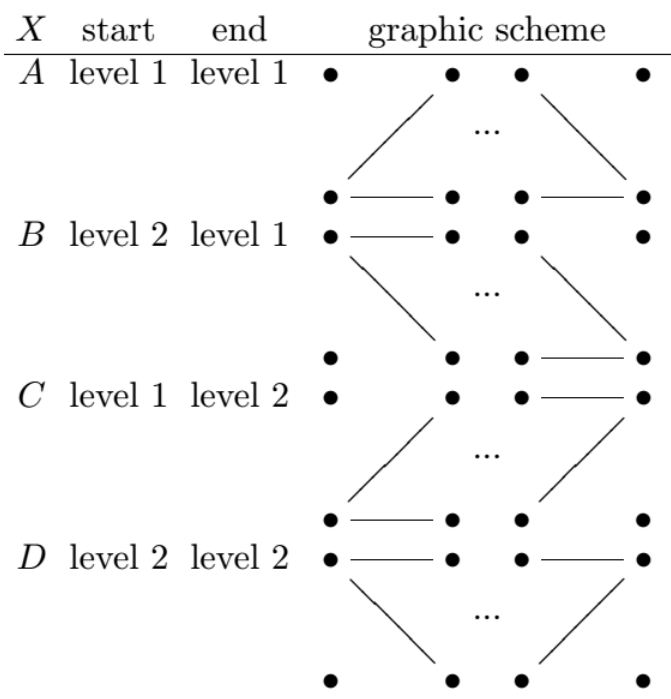

Fig. 3. Paths representing $\widehat{A}(\lambda), \widehat{B}(\lambda), \widehat{C}(\lambda), \widehat{D}(\lambda)$.

Quasi-continuity of paths provides all necessary and very simple rules of choice, for example: allowed monomial must have $\widehat{c}$ between two operators $\widehat{b}$, or $\widehat{a}$ must be linked with $\widehat{d}$ by appropriate operator $\widehat{b}$ or $\widehat{d}$.

The action of $m(\lambda)$ on arbitrarily chosen magnetic configuration $\left|f_{a}\right\rangle$ reads 


$$
m(\lambda)\left|f_{a}\right\rangle=\left\{\begin{array}{c}
0, \text { if } \widetilde{N}^{+} \cap \widetilde{N}_{c} \neq \varnothing \\
\quad \text { or } \widetilde{N}^{-} \cap \widetilde{N}_{b} \neq \varnothing \\
\mathrm{i}^{w_{b}+w_{c}} p^{u} q^{v}\left|f_{a}^{\prime}\right\rangle \text { otherwise }
\end{array}\right.
$$

where disjoint decompositions $\tilde{N}=\tilde{N}_{a} \cup \tilde{N}_{b} \cup \tilde{N}_{c} \cup \tilde{N}_{d}$ and $\tilde{N}=\tilde{N}^{+} \cup \tilde{N}^{-}$are defined by $\widehat{m}$ and $\left|f_{a}\right\rangle$, respectively. $\tilde{N}_{x}$ is the set of nodes on which $\widehat{m}$ is acting via the Lax object $\widehat{x} . \tilde{N}^{+}$and $\tilde{N}^{-}$are sets of nodes in which $\left|f_{a}\right\rangle$ has spins + and - . Therefore exponents $u, v$ are given by

$$
\begin{aligned}
& u=\left|\tilde{N}^{+} \cap \tilde{N}_{a}\right|+\left|\tilde{N}^{-} \cap \tilde{N}_{d}\right|, \\
& v=\left|\tilde{N}^{+} \cap \tilde{N}_{d}\right|+\left|\tilde{N}^{-} \cap \tilde{N}_{a}\right| .
\end{aligned}
$$

Equation (19) has interesting consequences, i.e.

- if $\widehat{m}$ and $\widehat{m}^{\prime}$ are different and the action of at least one of them on $\left|f_{a}\right\rangle$ is nonzero then

$$
m(\lambda)|f a\rangle \neq m^{\prime}(\lambda)|f a\rangle,
$$

which implies that each matrix element of $\widehat{M}$ is represented by only one monomial $\widehat{m}$.

- requirement for non-zero action with properties (16)(18) yields strict bounds on the numbers $w_{b}$ and $w_{c}$.

Therefore it is convenient to classify monomials with respect to the numbers $w_{b}$ or $w_{c}$ :

$$
\begin{aligned}
& M_{A r}= \\
& \bigcup_{m \in M_{A}}\left\{m \mid w(m)=\left(w_{a}, r, r, N-w_{a}-2 r\right)\right\}, \\
& M_{B r}= \\
& \bigcup_{m \in M_{B}}\left\{m \mid w(m)=\left(w_{a}, r, r-1, N-w_{a}-2 r+1\right)\right\}, \\
& M_{C r}= \\
& \bigcup_{m \in M_{C}}\left\{m \mid w(m)=\left(w_{a}, r-1, r, N-w_{a}-2 r+1\right)\right\}, \\
& M_{D r}= \\
& \bigcup_{m \in M_{D}}\left\{m \mid w(m)=\left(w_{a}, r, r, N-w_{a}-2 r\right)\right\} .
\end{aligned}
$$

There are fulfilled the following summation rules:

$$
\begin{aligned}
& \left|M_{A r}\right|=\left|M_{D r}\right|=\left(\begin{array}{l}
N \\
2 r
\end{array}\right), \\
& \left|M_{B r}\right|=\left|M_{C r}\right|=\left(\begin{array}{c}
N \\
2 r-1
\end{array}\right), \\
& \sum_{r=0}^{[N / 2]}\left(\begin{array}{l}
N \\
2 r
\end{array}\right)=2^{N-1}, \\
& \sum_{r=1}^{[N / 2]+1}\left(\begin{array}{c}
N \\
2 r-1
\end{array}\right)=2^{N-1},
\end{aligned}
$$

Another important classification comes from the fact that relation (19) associates $r_{0}$ of $r_{s}$ of spins $-1 / 2$ with the Lax objects $\widehat{b}$, therefore $r_{s}-r_{0}$ remaining ones can be freely distributed over arbitrarily accessible nodes. That allows to compute the number of nonzero matrix elements $\widehat{m}$ in the sector with $r_{s}$ spins $-1 / 2$ for the all of operators $\widehat{A}, \widehat{B}, \widehat{C}, \widehat{D}$, as

$$
\begin{gathered}
Z\left(m \mid r_{s}\right)=\left(\begin{array}{c}
N-2 r_{0} \\
r_{s}-r_{0}
\end{array}\right), \\
m \in M_{A r_{0}} \text { or } m \in M_{D r_{0}} \\
Z\left(m \mid r_{s}\right)=\left(\begin{array}{c}
N-2 r_{0}+1 \\
r_{s}-r_{0}
\end{array}\right), \\
m \in M_{B r_{0}} \text { or } m \in M_{C r_{0}} .
\end{gathered}
$$

\section{Conclusions}

Combinatorics of the Lax operators is a simple and convenient way of calculating monodromy matrix. Therefore it provides very simple algorithm for generation of the Bethe eigenvectors. Arbitrariness of distribution of the Bethe pseudoparticles over the set of nodes is the formal consequence of nonlocality of the model.

\section{References}

[1] H. Bethe, Z. Phys. 71, 205 (1931) (in German English translation in: D.C. Mattis, The Many-Body Problem, World Sci., Singapore 1993, p. 689.

[2] L.D. Faddeev, L.A. Takhtajan, LOMI 109, 134 (1981) (in Russian - English translation: J. Sov. Math. 24, 241 (1984)).

[3] L.A. Faddeev, arXiv:hep-th/9605187v1.

[4] J. Milewski, G. Banaszak, T. Lulek, M. Labuz, Physica B 406, 520 (2011).

[5] J. Milewski, B. Lulek, T. Lulek, M. Łabuz, R. Stagraczyński, Physica B 434, 14 (2014).

[6] J. Milewski, G. Banaszak, T. Lulek, M. Labuz, R. Stagraczyński, Open Syst. Inf. Dyn. 19, 1250012 (2012).

[7] T. Deguchi, P.R. Giri, arXiv:1408.7030, 2014.

[8] P.R. Giri, T. Deguchi, arXiv:1411.5839, 2014.

where $[N / 2]$ denotes integer part of $N / 2$. 